\title{
Sialólito em Artibeus lituratus (Olfers, 1818) (Chiroptera: Phyllostomidae): relato de caso
}

\author{
Eveline de Cássia Batista de Almeida Alves ${ }^{1 *}$ \\ Maria Juliana Gomes Arandas ${ }^{2}$ \\ Francisco Carlos Amanajás de Aguiar-Júnior ${ }^{3}$ \\ Katharine Raquel Pereira dos Santos ${ }^{3}$

\begin{abstract}
${ }^{1}$ Universidade Federal Rural de Pernambuco, Departamento de Ciência Veterinária Rua Dom Manoel de Medeiros, s/n, Dois Irmãos, CEP 52171-900, Recife - PE, Brasil ${ }^{2}$ Universidade Federal Rural de Pernambuco, Departamento de Morfologia e Fisiologia Animal ${ }^{3}$ Universidade Federal de Pernambuco, Centro Acadêmico de Vitória, Núcleo de Biologia Rua Alto do Reservatório, s/no, Bela Vista, CEP 55.608-680, Vitória de Santo Antão - PE, Brasil

* Autor para correspondência

evelinecassia27@hotmail.com
\end{abstract}

Submetido em 14/03/2015

Aceito para publicação em 12/08/2015

\section{Resumo}

Sialólitos são estruturas calcificadas que se desenvolvem dentro do sistema ductal salivar, apresentando formato redondo ou oval e, geralmente, são assintomáticos. Resultam da deposição de sais de cálcio ao redor de áreas focais de matéria orgânica e crescem continuamente, podendo provocar obstrução e redução do fluxo salivar. Comumente relatada em humanos, os sialólitos também podem, em menor frequência, acometer glândulas salivares de animais domésticos e silvestres. O trabalho objetivou descrever as características histopatológicas de um sialólito acometendo o ducto excretor de glândula salivar de um espécime adulto, macho, do morcego tropical Artibeus lituratus (Chiroptera: Phyllostomidae). Esse espécime foi coletado na área urbana de Vitória de Santo Antão (PE). Preparados histológicos foram corados com hematoxilina e eosina (HE) e técnicas histoquímicas com ácido periódico de Schiff (PAS) e azul de Alcian ( $\mathrm{pH}$ 1,0 e 2,5) foram aplicadas para melhor caracterização e descrição do sialólito. Embora a formação de sialólitos seja muito comum em glândulas salivares de mamíferos, sua ocorrência em morcegos ainda não havia sido relatada.

Palavras-chave: Glândula salivar; Morcego; Sialólitos

\section{Abstract}

Sialolith in Artibeus lituratus (Olfers, 1818) (Chiroptera: Phyllostomidae): case report. Sialoliths are calcified structures that develop into the salivary duct system, they have a round or oval shape and are usually asymptomatic. They result from the deposition of calcium salts around focal areas of organic matter and grow continuously, with the possibility of causing obstruction and reduced salivary flow. Commonly reported in humans, sialoliths may also affect, less frequently, the salivary glands of domestic and wild animals. This paper aimed to describe the histopathological characteristics of a sialolith affecting the excretory duct in the salivary gland of an adult male specimen of the tropical bat Artibeus lituratus (Chiroptera: Phyllostomidae). This specimen was collected in the urban area of Vitória de Santo Antão, Pernambuco, Brazil. Histological preparations were 
stained with hematoxylin and eosin (HE) and histochemical techniques with Schiff-periodic acid (PAS) and Alcian blue ( $\mathrm{pH} 1.0$ and 2.5) were applied for a better characterization and description of the sialolith. Although the formation of sialoliths is very common in the salivary glands of mammals, its occurrence in bats had not been reported before.

Key words: Bat; Salivary glands; Sialoliths

\section{Introdução}

Sialólitos são cálculos localizados nas glândulas salivares ou em seu ducto excretor, impedindo a secreção total ou parcial da saliva (NEVILLE et al., 2009) e caracterizam-se por estruturas calcificadas que apresentam formato oval ou esférico, de coloração amarelada à parda, com superfície lisa ou irregular, podendo ser únicos ou múltiplos (BAURMASH, 2004). O tamanho pode variar de microscópico a vários centímetros em seu maior eixo (ALCURE et al., 2005b). Estas estruturas são formadas por componentes orgânicos oriundos da saliva como glicoproteínas, mucopolissacarídeos, lipídios e restos celulares e de compostos inorgânicos como o fosfato de cálcio e carbonato de cálcio (ZEDEBSKI, 2003).

Ocorrem com maior frequência no interior e nos ductos das glândulas submandibulares, no entanto, podem acometer as parótidas, sublinguais, e glândulas salivares menores do palato, lábios e língua (ALCURE et al., 2005a). Normalmente, associado aos sialólitos, há um aumento volumétrico na região do ducto ou do corpo da glândula acometida, entretanto, a maioria dos casos é assintomática e de evolução lenta (BAURMASH, 2004; MACLEAN, 2006).

Artibeus lituratus, a espécie acometida com esta sialadenopatia, pertence à família Phyllostomidae e é considerado um morcego com ampla distribuição geográfica, sendo encontrado em quase todos os países, sobretudo no Brasil, com inúmeros registros em ambientes antropizados, possuindo hábito predominantemente frugívoro, porém pode incluir outros itens a sua dieta, bem como: recursos florais (néctar e pólen), folhas e insetos (PERINI et al., 2003; UIEDA; CHAVES, 2005).

Os sialólitos já foram relatados em humanos, porém há poucos registros de outros mamíferos acometidos com essa patologia, como: cães (PIGNONE et al.,
2009), gatos (SPANGLER; CULBERTSON, 1991), chimpanzés (ORKIN; BRASWELL, 1990), búfalos (JOSHI et al., 2003) e equinos (MACLEAN, 2006), entretanto para morcegos, ainda não havia registro de sialolitíase. Portanto, o presente estudo visa relatar a primeira ocorrência de sialólitos em glândula salivar menor do morcego neotropical A. lituratus.

\section{Material e Métodos}

Um espécime de A. lituratus, macho, adulto, pesando cerca de $60 \mathrm{~g}$, coletado na área urbana do município de Vitória de Santo Antão/PE (0807'05'S e $\left.35^{\circ} 17^{\prime} 29^{\prime \prime} \mathrm{W}\right)$, capturado por rede de neblina visando integrar o acervo de biodiversidade do Centro Acadêmico de Vitória (autorização do SISBIO nº 16070-1). Os espécimes capturados foram eutanasiados com dose letal de anestésico Xilazina ( $150 \mathrm{mg} / \mathrm{kg}$ ) na região peritonial, conforme normas do Comitê de Ética, autorização 23076.049197/2014-19.

As línguas foram extraídas após a retirada do crânio, tomando como referência o orifício epiglótico. Para identificação taxonômica da espécie foram realizadas análises da morfologia corporal, medida craniana, da fórmula dentária e análise morfológica dos caracteres linguais utilizando lupa estereoscópica.

Durante a análise taxonômica, foi evidenciada em um dos espécimes, leve intumescência na língua. Assim, o órgão foi coletado, clivado longitudinalmente e posteriormente submetido às técnicas histológicas de rotina; sendo desidratado em álcool etílico em concentrações crescentes, diafanizado em xilol, impregnados e incluídos em parafina. Os blocos obtidos foram cortados em micrótomo ajustado para $4 \mathrm{~mm}$. Sequencialmente, cortes seriados foram submetidos à técnica de coloração pela Hematoxilina-Eosina (H.E.), Ácido Periódico de Shifft (PAS) e Azul de Alcian pH 1,0 
e pH 2,5 e posteriormente, analisados em microscópio de luz (Nikon, E-200).

\section{Resultados}

Durante as análises histológicas da língua, a mucosa lingual (epitélio e lâmina própria) mostrou integridade, entretanto foi identificada na região anteroventral, a presença de estrutura arredondada, eosinofílica, de aproximadamente $0,5 \mathrm{~mm}$ de diâmetro (Figura 1A), formada por lamelas concêntricas apresentando um único centro de mineralização, com aglomerados amorfos nas porções mais periféricas e na região mais interna. Com base nessas características morfológicas foi estabelecido o diagnóstico de sialolitíase.

Visando a caracterização destes aglomerados quanto à presença de mucinas foram realizadas as colorações histoquímicas pelo ácido periódico de schiff (P.A.S), azul de alcian $\mathrm{pH}$ 2,5. Observou-se positividade por todos os corantes utilizados, no entanto, houve uma forte marcação pelo azul de alcian $\mathrm{pH} 2,5$ no interior e na periferia do sialólito (Figura 1B). O ducto glandular apresentou-se com ectasia e metaplasia escamosa e o tecido muscular estriado esquelético adjacente mostrouse atrofiado e infiltrado por células inflamatórias mononucleares, que também estavam presentes no tecido conjuntivo periductal (Figura 1C).

FIGURA 1: Fotomicrografia da língua de Artibeus lituratus. A - Crescimento gradual e um padrão lamelar observado no sialólito (seta), Hematoxilina e Eosina. B - Presença de mucinas ácidas não-sulfatadas (sialomucinas), Azul de alcian pH 2,5 (seta). C - Infiltrado inflamatório periductal moderado e de natureza crônica, Hematoxilina e Eosina; aumento de 400X.

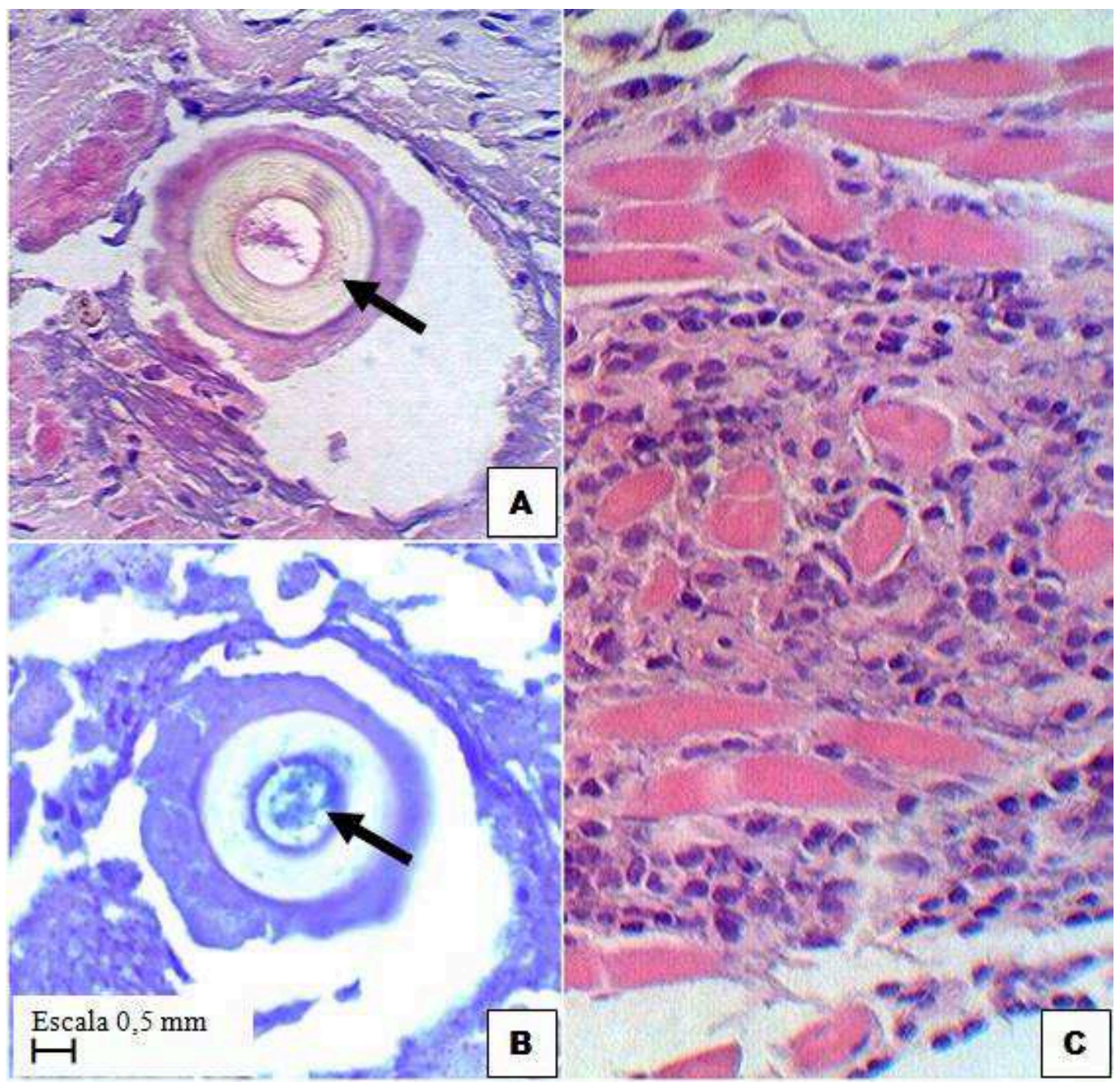




\section{Discussão}

Os cálculos salivares ou sialólitos possuem em sua composição, diferentes porções de matriz orgânica e inorgânica (ALCURE et al., 2005a; 2005b). Geralmente a parte orgânica se localiza no interior do cálculo, enquanto a periferia é formada essencialmente de elementos inorgânicos. No geral, as microanálises de sialólitos, através de raios-x revelam que os principais componentes inorgânicos são cálcio, fósforo, enxofre e sódio, sendo os dois primeiros encontrados na forma de cristais de hidroxiapatita (MIMURA et al., 2005).

Cerca de $80 \%$ dos sialólitos acometem a glândula submandibular e seu sistema ductal. Este fato se deve à composição salivar, além da predisposição anatômica à estagnação salivar ocasionada pelo ducto de Wharton, que é longo, apresenta um trajeto irregular, ascendente e um orifício estreito (LANDGRAF et al., 2006; TANDLER, 1993). No espécime de morcego acometido, a localização intraductal do sialólito na região antero-ventral da língua é compatível com a localização anatômica do ducto excretor da glândula lingual em algumas espécies de morcegos (GRIFFITHS; CRILEY, 1989).

As análises histoquímicas constataram a presença de mucina através das colorações P.A.S, azul de alcian pH 2,5 no interior do sialólito. A forte marcação pelo azul de alcian $\mathrm{pH}$ 2,5, indicou que havia grande quantidade de mucinas ácidas não-sulfatadas (sialomucinas) (MYERS et al., 2008) e, o acúmulo de mucinas salivares, pode ter influenciado o processo de calcificação, que resulta em uma deposição rítmica de cristais de hidroxiapatita, crescimento gradual e um padrão lamelar (ANNEROTH et al., 1978; ZEDEBSKI, 2003).

Foram observadas alterações morfológicas no ducto associado ao sialólito, como, ectasia e metaplasia escamosa, que comumente ocorrem nas obstruções e irritações crônicas do mesmo (NEVILLE et al., 2009). Além disso, observou-se um foco de infiltrado inflamatório periductal moderado e de natureza crônica, que pode estar associada à presença de bactérias, como um dos fatores etiológicos na formação de cálculos (LUSTMANN; SHTEYER, 1981), apesar desta etiologia, ter sido motivo de muitas discussões em outros estudos (ISACSSON; FRISKOPP, 1984).

Apesar da ocorrência comum em humanos, a etiologia dos sialólitos, permanece incerta, porém considerou-se que, em áreas urbanas, a baixa disponibilidade e qualidade dos alimentos de sua preferência podem levar a espécie A. lituratus diversificar sua dieta (NOVAES; NOBRE, 2009), este fator pode comprometer a produção e a composição da secreção das glândulas salivares, acarretando em mudanças do $\mathrm{pH}$, no fluxo e o aumento nas concentrações de cálcio salivar, que contribuem na precipitação de cálcio e também têm sido apontados como fatores importantes na formação de sialólitos (ASHBY, 1995).

O tratamento das obstruções provocadas por estes cálculos, nas diversas espécies de mamíferos acometidas, depende da presença de sintomas, sua duração, quantidade e tamanho dos sialólitos e sua localização (PIGNONE et al., 2009). Os sialólitos costumam ser únicos, e quando associados às glândulas salivares maiores, são removidos através de manipulação da glândula ou ducto e excisão cirúrgica (NEVILLE et al., 2009).

Os estudos sobre patologias salivares em mamíferos silvestres são escassos. Particularmente em morcegos, as informações sobre patologias orais se restrigem apenas às patologias dentárias, como a incidência de cáries, erosões e desgaste dentário e inflamações periodontais (PHILLIPS, 1971).

Os sialólitos sao comumente relatados em humanos, e ocorre em menor frequência em outras espécies de mamíferos, porém para morcegos ainda não haviam sido relatados. No presente caso, atribuiu-se a ocorrência da dessa patologia em A. lituratus, à alterações tróficas em áreas urbanas de Vitória de Santo Antão/PE, que possivelmente alterou a dieta e comprometeu a produção, a composição e o fluxo da secreção salivar no ducto excretor da glândula lingual do espécime em questão.

\section{Referências}

ASHBY, R. A. The chemistry of sialoliths: stones and their homes. In: NORMAN, J. E. D.; MCGURK, M. (Ed.). Color atlas and text of the salivary glands. Diseases, Disorders, and Surgery. London: Mosby-Wolfe, 1995. p. 243-251. 
ALCURE, M. L.; DELLA COLETTA, R.; GRANER, E.; HIPOLITO JR., O.; LOPES, M. A. Sialolithiasis of minor salivary glands: a clinical and histopathological study. Scandinavian Journal of Dental Research, Copenhagen, v. 53, n. 4, p. 27881,2005 b.

ALCURE, M. L.; VARGAS, P. A.; JORGE JÚNIOR, J.; DELlA COLETTA R.; DI HIPOLITO O. J.; LOPES M. A. Clinical and histopathological findings of sialoliths. Brazilian Journal of Oral Sciences, Piracicaba, v. 4, n. 15, p. 899-903, 2005a.

ANNEROTH, G.; ENEROTH, C. M.; ISACSSON, G.; LUNDQUIST, P. G. Ultrastructure of salivary calculi. Scandinavian Journal of Dental Research, Copenhagen, v. 86, n. 3, p. 182-192, 1978.

BAURMASH, H. Submandibular salivary stones: current management modalities. British Journal of Oral and Maxillofacial Surgery, London, v. 62, p. 369-378, 2004.

BHASKAR, S. N. Histologia e embriologia oral de Orban. 10 ed. São Paulo: Artes Médicas, 1989. 501 p.

GRIFFITHS, T. A.; CRILEY, B. B. Comparative lingual anatomy of the bats Desmodus rotundus and Lonchophylla robusta (Chiroptera: Phyllostomidae). Journal of Mammalogy, Lawrence, v. 70, n. 3, p. 608-613, 1989.

ISACSSON, G.; FRISKOPP. J. The morphology of salivary calculi: a scanning electron microscopic study. Acta Odontologica Scandinavica, Copenhagen, v. 42, n. 2, p. 65-72, 1984.

JOSHI, V. B.; TYAGI, S. P.; SHARMA, A. Obstructive Sialolithiasis in buffalo and its management. Buffalo Bulletin, Buffalo, v. 22, n. 1, p. 3-5, 2003.

LANDGRAF, H.; ASSIS, A. F.; KLÜPPEL, L. E.; OLIVEIRA, C. F.; GABRIELLI, M. A. C. Extenso sialólito no ducto da glândula submandibular: relato de caso. Revista de Cirurgia e Traumatologia Buco-Maxilo-Facial, Camaragibe, v. 6, n. 2, p. 29-34, 2006.

LUSTMANN, J.; SHTEYER, A. Salivary calculi: ultrastructural morfology and bacterial etiology. Scandinavian Journal of Dental Research, Copenhagen, v. 60, n. 8, p. 1386-1395, 1981.

MACLEAN, Y. T. Chronic sialolithiasis in a Trakehner mare. The Canadian Veterinary Journal, Ottawa, v. 47, n. 5, p. 480-482, 2006.

MIMURA, M.; TANAKA, N.; ICHINOSE, S.; KIMIJIMA, Y.; AMAGASA, T. Possible etiology of calculi formation in salivary glands: biophysical analysis of calculus. Medical Molecular Morfophology, Gifu, v. 38, n. 3, p. 189-95, 2005.
NEVILLE, B. W.; DAMM, D. D.; ALLEN, C. M.; BOUQUOT, J. E. Oral and maxillofacial pathology. 3. ed. St. Louis: Saunders Elsevier, 2009. 968 p.

NOVAES, R. L. M.; NOBRE, C. C. Dieta de Artibeus lituratus (Olfers, 1818) em área urbana na cidade do Rio de Janeiro: frugivoria e novo registro de folivoria. Chiroptera Neotropical, Brasília, v. 15, n. 2, p. 487-493, 2009.

MYERS, B. M.; FREDENBURGH, J. L.; GRIZZLE, W. E. Carbohydrates. In: BANCROFT, J. D.; GAMBLE, M. (Ed.). Theory and practice of histological techniques. Cap. 11. 6. ed. Philadelphia: Elselvier, 2008. p. 161-187.

ORKIN, J. L; BRASWELL, L. D. Sialolithiasis in two chimpanzees. Journal of the American Veterinary Medical Association, Schaumburg, v. 196, p. 1651-1653, 1990.

PHILLIPS, C. J. The dentition of glossophagine bats: development, morphological characteristics, variation, pathology, and evolution. Bulletin of the University of Kansas Museum of Natural History, Kansas City, v. 54, p. 1-138, 1971.

PERINI, F. A.; TAVARES, V. C.; NASCIMENTO, C. M. D. Bats from the city of Belo Horizonte, Minas Gerais, southeastern Brazil. Chiroptera Neotropical, Brasília, v. 9, p. 169-173, 2003.

PIGNONE, V. N.; FARACO, C. S.; ALBURQUERQUE, P. B.; RECLA, G.; GIANOTTI, G.; CONTESINI, E. A. Sialólito no ducto da glândula mandibular em cão. Acta Scientiae Veterinariae, Porto Alegre, v. 37, n. 3, p. 277-280, 2009.

SPANGLER, W. L.; CULBERTSON, M. R. Salivary gland disease in dogs and cats: 245 cases (1985-1988). Journal of the American Veterinary Medical Association, Schaumburg, v. 198, p. 465-469, 1991.

TANDLER, B. Structure of the duct system in salivary glands mammalian major. Microscopy Research and Technique, Malden, v. 26, p. 57-74, 1993.

UIEDA, W.; CHAVES, M. E. Bats from Botucatu region, state of São Paulo, Southern Brazil. Chiroptera Neotropical, Brasília, v. 11, p. 224-226, 2005.

ZEDEBSKI, R. A. M. Análise morfológica e estudo de sialólitos por meio das microscopias ópticas e de polarização. 2003. 125 f. Dissertação (Mestrado em Odontologia - Universidade de São Paulo, Bauru. 2003. 